\title{
Radical Robot-Assisted Liver Resection for Alveolar Echinococcosis
}

\author{
Mikhail Efanov, MD, PhD, Ruslanh Alikhanov, MD, PhD, Victor Cvirkun, MD, PhD, \\ Ivan Kazakov, MD, PhD, Olga Melekhina, MD, PhD, Pavel Kim, MD, Andrey Vankovich, MD, \\ Igor Khatkov, MD, PhD \\ Department of Hepato-pancreato-biliary Surgery, Moscow Clinical Scientific Center, Moscow, Russia (Drs. Efanov, \\ Alikhanov, Kazakov, Melekhina, Kim, and Vankovich and Prof. Cvirkun). \\ Moscow Clinical Scientific Center, Moscow, Russia (Prof. Khatkov).
}

\begin{abstract}
Introduction: Radical surgery is the only curative method for liver alveolar echinococcosis (AE). To date, there is no information about the efficacy of laparoscopy including robot-assisted liver resection in radical treatment of AE. This is a case report of a robot-assisted radical liver resection for $\mathrm{AE}$.

Case Description: A 51-year-old man was admitted to the clinic with complaints of weakness and pain in the right abdomen. Computed tomography (CT) revealed a lesion measuring $46 \times 52 \times 86 \mathrm{~mm}$, located in liver segments VI and VII, and robot-assisted resection of those segments was performed. The surgery lasted for 485 minutes, and intraoperative blood loss was $1000 \mathrm{~mL}$. The position of the patient, sites of trocars placement, and details of the surgery are described. The patient had an uneventful postoperative course and was discharged on day 10 . Albendazole, was administered for 2 years as antiparasitic chemotherapy. Dynamic monitoring for more than 13 months showed no recurrence of the disease.

Discussion: Publications on the use of laparoscopic and robotic resection of posterior liver segments are scarce, but experience has shown that the learning curve for the use of the robotic system is shortened in comparison with that for other approaches. After the case reported herein, we have performed similar procedures and have seen a marked decrease in blood loss and operative time. This case and our growing experience in performing radical robot-assisted liver resections demonstrate the feasibility of using robotic laparoscopic approaches for radical treatment of early diagnosed liver AE, particularly when the affected liver segment is difficult to reach.
\end{abstract}

Key Words: Alveolar echinococcosis, Hepatectomy, Liver resection, Radical treatment, Robotic surgical procedures

Citation Efanov M, Alikhanov R, Cvirkun V, Kazakov I, Melekhina O, Kim P, Vankovich A, Khatkov I. Radical robot-assisted liver resection for alveolar echinococcosis. CRSLS e2015.00021. DOI: 10.4293/CRSLS.2015.00021.

Copyright (c) 2015 by SLS, Society of Laparoendoscopic Surgeons. This is an open-access article distributed under the terms of the Creative Commons Attribution-Noncommercial-ShareAlike 3.0 Unported license, which permits unrestricted noncommercial use, distribution, and reproduction in any medium, provided the original author and source are credited.

Address correspondence to: Mikhail Efanov, MD, PhD, Department of Hepato-Pancreato-Biliary Surgery, Moscow Clinical Scientific Center 11123, Shosse Entuziastov, 86, Moscow, Russia. Telephone: +7 (916) 105-88-30, Fax: +7 (495) 304-19-42, E-mail: m.efanov@mknc.ru

\section{INTRODUCTION}

Alveolar echinococcosis (AE) is a rare parasitic disease that is usually diagnosed late, making radical surgery unfeasible in all patients. ${ }^{1}$

The published literature contains no information regarding the possibility of using laparoscopic methods in the radical treatment of AE. In this regard, we present our own observation of a robot-assisted resection of segments VI and VII in a liver affected by AE, with a 1-year follow-up of the postoperative outcome.

\section{CASE REPORT}

A 51-year-old man, who worked as a long-haul truck driver, was admitted to the hospital in November 2013, reporting weakness, recurrent pain in the right abdomen unrelated to food intake, and periodic episodes of temperature elevated to 


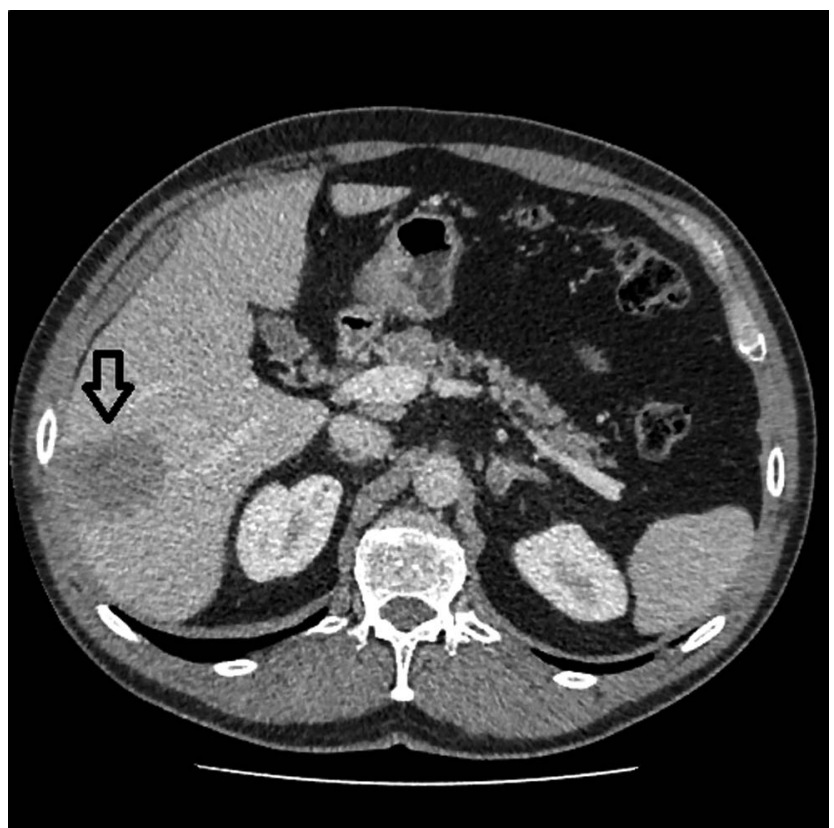

Figure 1. CT axial scan, venous phase. A lesion was located in liver segments VI and VII (arrow).

subfebrile levels. The symptoms had persisted since early 2013. In March 2013, a mass was found in the right lobe of the liver during an examination prompted by the symptoms.

Based on the computed tomographic (CT) data, a lesion measuring $46 \times 52 \times 86 \mathrm{~mm}$ was revealed in liver segments VI and VII, with an uneven and unclear outline, an inhomogeneous structure with an area of cavitation, and poor uptake of the contrast agent. The CT-identified mass in the right lobe required morphologic verification (Figures 1, 2).

Histologic examination of the material collected by percutaneous needle biopsy of the mass revealed signs of inflammation and an area of necrosis. No tumor elements were detected. Endoscopy and colonoscopy revealed no organic changes. The levels of the tumor markers $\alpha$-fetoprotein (AFP), carcinoembryonic antigen (CEA), and cancer antigen (CA) 19-9 were within normal range. A qualitative test for the presence of antibodies to echinococcus was positive.

In November 2013, robot-assisted resection was performed on liver segments VI and VII, with the use of the da Vinci Si Surgical System (Intuitive Surgical Systems, Sunnyvale, California) robotic cell. The patient was positioned supine, with the head of the table elevated to $30^{\circ}$ and the table tilted $30^{\circ}$ to the left. After pneumoperitoneum was established, the trocar for the camera was inserted $6 \mathrm{~cm}$ to the right of the umbilicus. Two robotic

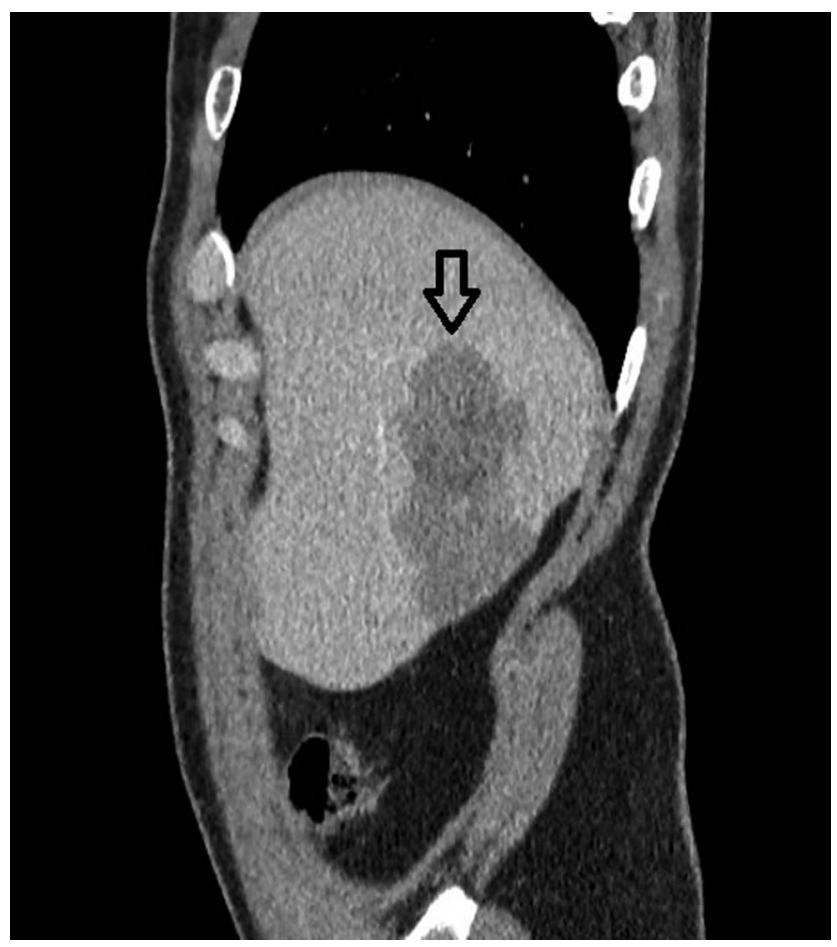

Figure 2. CT sagittal scan, venous phase. A lesion was located in liver segments VI and VII (arrow).

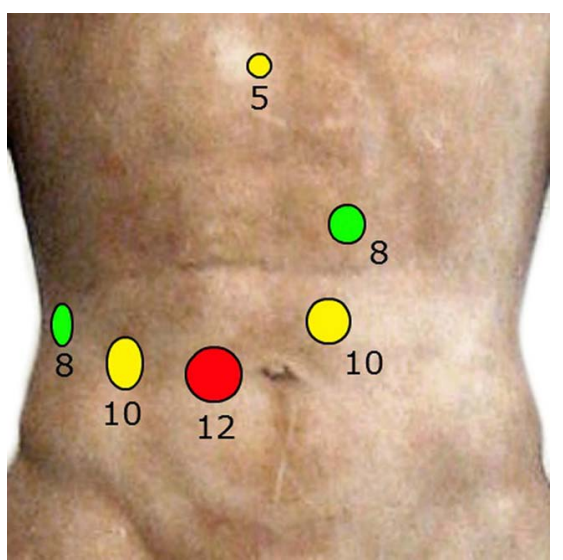

Figure 3. The placement of the ports (scheme). The positions of the trocars are marked by different colors: trocar for the camera, red; robotic trocars, green; and auxiliary ports, yellow.

trocars were introduced: the first in the right upper quadrant, $4 \mathrm{~cm}$ below the costal arch along the anterior axillary line, and the second in the epigastrium, $8 \mathrm{~cm}$ below the xiphoid process and $4 \mathrm{~cm}$ to the left of the midline. Three auxiliary ports were set up between the robotic instrument and the camera ports. The placement of the ports is shown in Figure 3. 


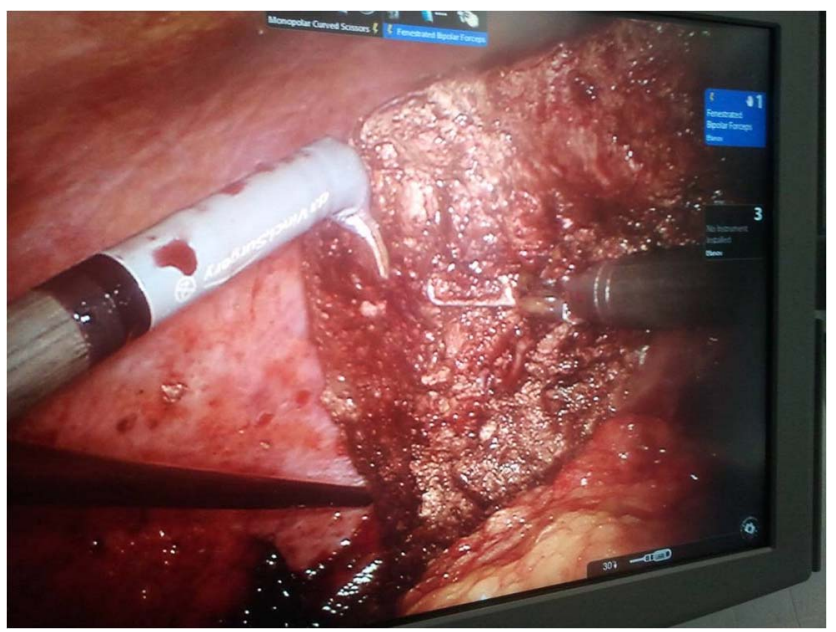

Figure 4. Final view of the operative field. Robotic scissors and fenestrated bipolar clamp shifted the liver to the left, opening the resection surface.

On examination of the abdominal cavity, the parasitic lesion could not be visualized because of the mainly intrahepatic location of the node and its localization in the right posterior section. Examination of the abdominal cavity, including the right dome of the diaphragm, revealed no extrahepatic abdominal lesions. The right lobe of the liver was fully mobilized; the short hepatic veins were not cut. A tourniquet was applied to the hepatoduodenal ligament.

Liver dissection was performed with 2 robotic instruments: a harmonic ultrasonic scalpel, adapted for use with the robotic manipulator, and a bipolar Maryland dissector. Because the parasitic tissue node, due to its large size, was squeezing the anterior section, the resection plane was shifted to the right. The Glissonian pedicles to segments VI and VII and the right hepatic vein branches that drain these segments were isolated, ligated, and clipped in the process of dissecting the parenchyma of the liver. The trunk of the right hepatic vein was not cut. The final view of the operating field is shown in Figure 4 .

The parasitic node was removed from within healthy tissue. The minimum width of free surgical margin was 5 $\mathrm{mm}$. The removed specimen is shown in Figures 5 and $\mathbf{6}$. The resected liver fragment with the parasitic node was placed in a plastic container and removed through a $6-\mathrm{cm}$ Pfannenstiel laparotomy incision.

The surgery lasted for 485 minutes, with intraoperative blood loss of $1000 \mathrm{~mL}$. An intermittent Pringle maneuvre was used for up to 15 minutes, with pauses of at least 5

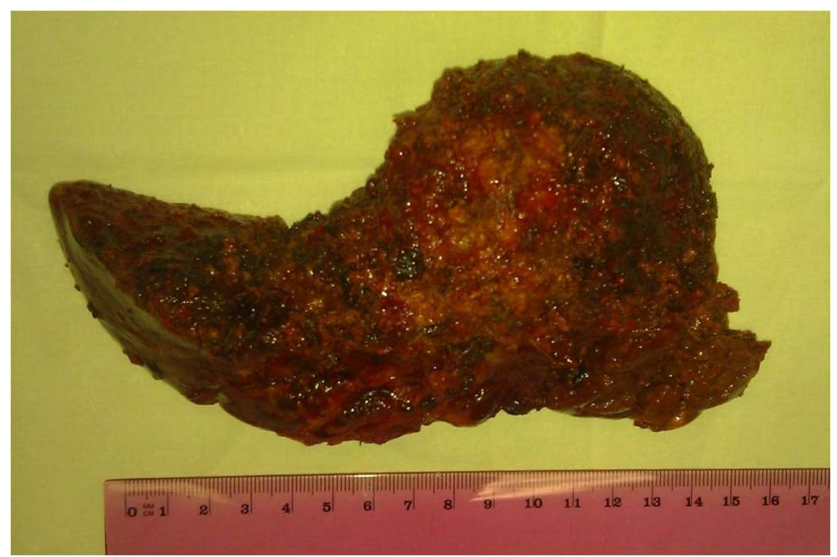

Figure 5. Resected specimen. The parasitic node was removed from healthy tissue.

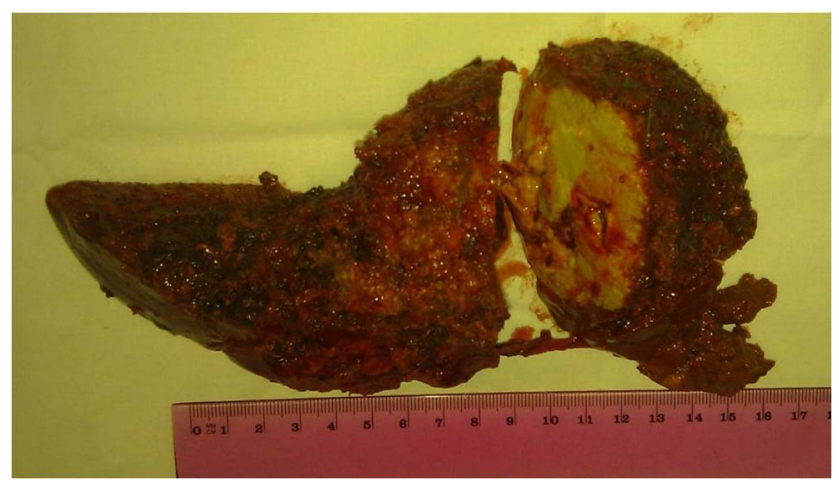

Figure 6. Resected specimen. Solid parasitic tissue with cavitation area on the cut surface. The minimum width of free surgical margin was $5 \mathrm{~mm}$.

minutes. The postoperative course was uneventful, and the patient was discharged on day 10.

Histologic examination revealed a metacestode that presented as a multivesicular structure consisting of numerous aggregated small cysts, not exceeding a few millimeters in diameter (Figure 7).

Antiparasitic chemotherapy with Albendazole was administered for 2 years. The patient remained under dynamic monitoring for more than 13 months, during which no recurrence was observed.

\section{DISCUSSION}

$\mathrm{AE}$ is a zoonotic disease found only in the Northern hemisphere. The causative agent of the disease in humans is the parasite at the larval stage of development. The spread of the disease is associated with habitats of the principal hosts of the parasite: red foxes and raccoons. ${ }^{2}$ 


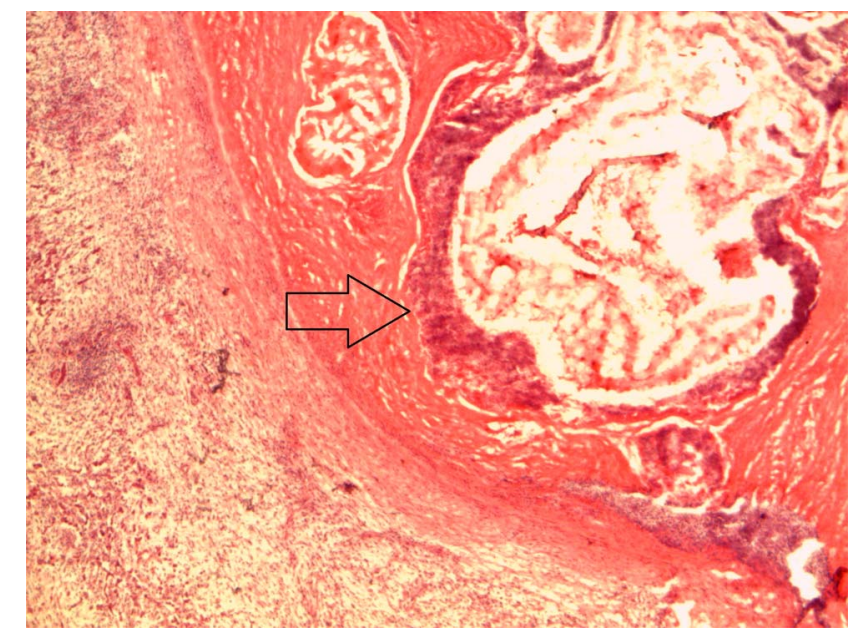

Figure 7. Histologic examination of resected specimen. Hematoxylin and eosin stain of paraffin-embedded slices. Alveolar cysts with an irregular wall and peripheral formation of granulation tissue (arrow). Magnification, $\times 200$.

The annual incidence ranges from 0.03 to 1.2 per 100,000 people. ${ }^{3}$ The most commonly affected organ is the liver, mainly in the right lobe. ${ }^{1}$ The size of the parasitic node increases because of the infiltrative growth, resembling a malignant tumor. ${ }^{2,4}$ Symptoms of the disease usually develop in patients with locally advanced tumor growth.

The diagnosis is based on a combination of the clinical presentation, pertinent history, changes detected by radiological techniques, and positive serologic tests. Because of its latent course, AE is usually diagnosed late. The disease often manifests as complications caused by locally advanced disease. Most of the patients at the time of initial diagnosis exhibit stage 3-4 of the disease (ie, locally advanced or extrahepatic lesion or both). ${ }^{5}$

Treatment involves a multidisciplinary approach. Preference is given to radical surgical treatment, involving R0 resection. ${ }^{2}$ Nonradical resections (R1/2) are not recommended, as they do not provide a survival advantage when compared with long-term conservative treatment. ${ }^{6}$

A search of the PubMed and Scopus databases with the keywords alveolar echinococcosis, laparoscopic, robotic, and liver resection found no publications devoted to performing the surgery, either with robotic devices or by laparoscopy. Laparoscopic intervention with alveococcosis of the liver is mentioned in one publication as a diagnostic method.?

According to review articles published in 2013, robotic liver resection was used in 2 of 3 of the observed patients to treat liver tumors and in 1 of 3 to treat various benign focal lesions. ${ }^{8,9}$ At present, Giulianotti et $\mathrm{a}^{8}$ have reported the most experience with robotic resections of the liver.

The specific feature of this observation is not only that it demonstrates successful robotic intervention, but also early diagnosis of the disease at stage I (P1NOM0), which defines the possibility of radical surgery.

Another feature of this operation was the fully laparoscopic robot-assisted segment-oriented resection of liver segments VI and VII. To date, there is a lack of publications dedicated to analysis of laparoscopic and robotic resection of posterior liver segments. ${ }^{10-13}$ The lesions in segments VII and VIII more commonly are resected via a laparoscopic right hepatectomy, sacrificing a substantial volume of normal liver, because laparoscopic resection of superoposterior segments is particularly challenging. ${ }^{14,15}$ The undeniable advantages of robotic systems include the high degree of flexibility of robotic tools, the ability of the surgeon to control the camera, and high image stability. The aggregate benefits of the robotic system can dramatically shorten the learning curve. ${ }^{14}$ The use of robotic technologies is one of the evident strategies for optimizing the parenchyma-sparing resection of superoposterior liver segments. ${ }^{16,17}$ Tranchart et al ${ }^{17}$ reported that more tumors were localized in the superior and posterior segments in patients who underwent robot-assisted liver resection than in whose who underwent the traditional laparoscopic approach.

The second International Consensus Conference of Laparoscopic Liver Resection, held in Iwate, Japan, in October 2014, recommended using a point-based scoring system to assess the difficulty of laparoscopic liver resection. It is not recommended that liver resections with scores above 10 points be performed by laparoscopic methods. ${ }^{18}$ The presented operation scored 9.452 points, which puts it in the category of laparoscopic resections of high difficulty. Our overall experience from November 2013 to December 2014 included 16 robot-assisted liver resections with an average difficulty index of $8 \pm 1.68$ (range, 4.3-10.2) (unpublished data).

Our initial experience, including the case presented in this article, testifies to the possibility of safely performing robotassisted liver resections of a high difficulty index in the absence of substantial experience with similar interventions by classic laparoscopic methods. In our experience, in most of the resections performed, the focal lesions were localized in the posterior segments of the liver (69\%), including liver resections performed in 7 patients due to involvement of liver segments VI and VII (unpublished data). 
The case presented herein was our first robotic resection of posterior liver segments, with no experience in using a laparoscopic approach in this procedure. That is why the blood loss was relatively high in comparison with the loss in similar procedures performed later. The mean blood loss was $575 \pm 462 \mathrm{~mL}$, and the mean time was $495 \pm 83$ minutes in our later posterior liver segments resections (unpublished data). The mean blood loss during laparoscopic posterior liver segments resection can rise to 1100 $\mathrm{mL}$. The mean operative time, even in expert hands, can reach 344 minutes (range, 99-685). ${ }^{10}$ Technical improvement depends on the learning curve, which usually includes not less than 20-30 similar procedures in laparoscopic liver surgery. ${ }^{19}$

\section{CONCLUSION}

The presented case of radical robot-assisted liver resection demonstrates the feasibility of using laparoscopic procedures for radical treatment of $\mathrm{AE}$ of the liver, subject to early diagnosis. The da Vinci robotic system is an effective tool that can be used in cases where the posterior segments are involved and there is little surgical experience in classic laparoscopic liver resection.

\section{References:}

1. Kern P, Bardonnet R, Renner E, et al. European Echinococcosis Registry: Human Alveolar Echinococcosis, Europe, 19822000. Emerg Infect Dis. 2003;9:343-349.

2. Brunettia E, Kernb P, Vuittonc DA. Expert consensus for the diagnosis and treatment of cystic and alveolar echinococcosis in humans. Acta Trop. 2010;114:1-16.

3. Schweiger A, Ammann RW, Candinas D, et al. Human alveolar echinococcosis after fox population increase, Switzerland. Emerg Infect Dis. 2007;13:878-882.

4. Ammann RW, Eckert J. Cestodes: echinococcus [published correction appears in Gastroenterol Clin North Am. 1996;25:vii]. Gastroenterol Clin North Am. 1996;25:655-689.

5. Nahorski WL, Knap JP, Pawłowski ZS, et al. Human alveolar echinococcosis in Poland: 1990-2011. PLoS Negl Trop Dis. 2013; $7: 1-8$.

6. Buttenschoen K, Carli Buttenschoen D, et al. Long-term experience on surgical treatment of alveolar echinococcosis. Langenbecks Arch Surg. 2009;394:689-698.
7. Delbecque K, Detry O, Hayette MP, et al. A case of hepatic alveolar echinococcosis contracted in Belgium. Acta Gastroenterol Belg. 2002;65:55-60.

8. Giulianotti PC, Coratti A, Sbrana F, et al. Robotic liver surgery: results for 70 resections. Surgery. 2011;149:29-39.

9. Reggiani P, Antonelli B, Rossi G. Robotic surgery of the liver: Italian experience and review of the literature. Ecancermedicalscience. 2013;26:358.

10. Ikeda T, Toshima T, Harimoto N, et al. Laparoscopic liver resection in the semiprone position for tumors in the anterosuperior and posterior segments, using a novel dual handling technique and bipolar irrigation system. Surg Endosc. 2014;28: $2484-2492$.

11. Kazaryan AM, Røsok BI, Marangos IP, et al. Comparative evaluation of laparoscopic liver resection for posterosuperior and anterolateral segments. Surg Endosc. 2011;25:3881-3889.

12. Cho JY, Han HS, Yoon YS, et al. Outcomes of laparoscopic right posterior sectionectomy in patients with hepatocellular carcinoma in the era of laparoscopic surgery. Surgery. 2015; 19.pii: S0039-606000092-6.

13. Coles SR, Besselink MG, Serin KR, et al. Total laparoscopic management of lesions involving liver segment 7. Surg Endosc. In press.

14. Leung U, Fong Y. Robotic liver surgery. Hepatobiliary Surg Nutr. 2014;3:288-294.

15. Cho JY, Han HS, Yoon YS, et al. Feasibility of laparoscopic liver resection for tumors located in the posterosuperior segments of the liver, with a special reference to overcoming current limitations on tumor location. Surgery. 2008;144:32-38.

16. Casciola L, Patriti A, Ceccarelli G, et al. Robot-assisted parenchymal-sparing liver surgery including lesions located in the posterosuperior segments. Surg Endosc. 2011;25:3815-3824.

17. Tranchart H, Ceribelli C, Ferretti S, Dagher I, Patriti A. Traditional versus robot-assisted full laparoscopic liver resection: a matched-pair comparative study. World J Surg. 2014;38:29042909.

18. Ban D, Tanabe M, Ito $\mathrm{H}$, et al. A novel difficulty scoring system for laparoscopic liver resection. J Hepatobiliary Pancreat Sci. 2014;21:745-753.

19. Cai X, Li Z, Yu H, et al. Laparoscopic liver resection and the learning curve: a 14-year, single-center experience. Surg Endosc. 2014;28:1334-1341. 